\title{
Real-Time and Low-Power Streaming Source Separation Using Markov Random Field
}

\author{
GLENN G. KO and ROB A. RUTENBAR, University of Illinois at Urbana-Champaign
}

\begin{abstract}
Machine learning (ML) has revolutionized a wide range of recognition tasks, ranging from text analysis to speech to vision, most notably in cloud deployments. However, mobile deployment of these ideas involves a very different category of design problems. In this article, we develop a hardware architecture for a sound source separation task, intended for deployment on a mobile phone. We focus on a novel Markov random field (MRF) sound source separation algorithm that uses expectation-maximization and Gibbs sampling to learn MRF parameters on the fly and infer the best separation of sources. The intrinsically iterative algorithm suggests challenges for both speed and power. A real-time streaming FPGA implementation runs at $150 \mathrm{MHz}$ with $207 \mathrm{~KB}$ RAM, achieves a speed-up of $22 \times$ over a software reference, performs with an SDR of up to $7.021 \mathrm{~dB}$ with $1.601 \mathrm{~ms}$ latency, and exhibits excellent perceived audio quality. A 45nm CMOS ASIC virtual prototype simulated at $20 \mathrm{MHz}$ shows that this architecture is small ( $<10$ million gates) and consumes only $70 \mathrm{~mW}$, which is less than $2 \%$ of the power of an ARM Cortex-A9 software version. To the best of our knowledge, this is the first Gibbs sampling inference accelerator designed in conventional FPGA/ASIC technology that targets a realistic mobile perceptual application.
\end{abstract}

CCS Concepts: • Computing methodologies $\rightarrow$ Learning in probabilistic graphical models; $\bullet$ Hardware $\rightarrow$ Hardware accelerators; Application specific integrated circuits;

Additional Key Words and Phrases: Real-time streaming, blind source separation, Markov random field, maximum a posteriori inference, Gibbs sampling

\section{ACM Reference format:}

Glenn G. Ko and Rob A. Rutenbar. 2018. Real-Time and Low-Power Streaming Source Separation Using Markov Random Field. F. Emerg. Technol. Comput. Syst. 14, 2, Article 17 (May 2018), 22 pages.

https://doi.org/10.1145/3183351

\section{INTRODUCTION}

We are living in an era where artificial intelligence in general, and machine learning (ML) in particular, is making great practical strides. This explosion of ML has been enabled in part by the growth of economical computing power available to researchers to deploy these techniques widely. Multi-core CPUs, GPUs, and even FPGAs have all provided significantly cheaper computing resources and, paired with cheaper storage devices, allowed many researchers to try complex and sophisticated algorithms trained on vast datasets. The most notable examples are deep neural

This work was supported in part by Systems on Nanoscale Information fabriCs (SONIC), one of the six SRC STARnet Centers, sponsored by MARCO and DARPA, and the National Science Foundation under grant No. CCF-1302641.

Authors' addresses: G. G. Ko, Harvard University, 33 Oxford Street, Cambridge, MA 02138; email: gko@seas.harvard.edu; R. A. Rutenbar, 132 Cathedral of Learning, University of Pittsburgh, Pittsburgh, PA 15260; email: rutenbar@pitt.edu.

Permission to make digital or hard copies of all or part of this work for personal or classroom use is granted without fee provided that copies are not made or distributed for profit or commercial advantage and that copies bear this notice and the full citation on the first page. Copyrights for components of this work owned by others than ACM must be honored. Abstracting with credit is permitted. To copy otherwise, or republish, to post on servers or to redistribute to lists, requires prior specific permission and/or a fee. Request permissions from permissions@acm.org.

(C) 2018 ACM 1550-4832/2018/05-ART17 \$15.00

https://doi.org/10.1145/3183351 
networks (DNNs) and their application, first, to image classification and then to a wide array of other tasks. While the concept of neural networks has been around for decades, the breakthrough of the "deep" formulations coupled with relatively efficient GPU-based deployment (Krizhevsky et al. 2012), made for visible successes such as the large-scale ImageNet competition (Russakovsky et al. 2015) won by a DNN by a large margin over shallow ML methods. More recent work has shown accuracy levels close to or even better than that of humans (He et al. 2015). As a consequence of these successes, there has been a tremendous amount of research, in both academia and industry, to accelerate DNNs via custom hardware architectures using GPUs, FPGAs, and ASICs. Most of these efforts examine architectures suitable for enterprise deployments, e.g., in cloud data centers.

However, DNNs are not the only interesting and practical platform for building useful ML applications, and large-scale enterprise deployments are not the only places we might want to deliver hardware accelerators that render these methods. DNNs, where (loosely speaking) the basic compute kernel is matrix multiplication, happen to fit modern GPU architectures quite well, and so their deployment in cloud scenarios was, perhaps, inevitable. However, there are other widely used and powerful machine-learning algorithms that do not fit well on conventional GPU platforms. In this work, we focus on one broad category of ML tasks: probabilistic graphical models (PGMs) (Koller and Friedman 2009). A PGM is a large graph where, roughly, nodes represent what we know and what we observe (think: random variables) and edges represent relationships among these observables (think joint or conditional probability distributions). The process of asking questions of these graphs, such as "what is the most likely set of labels for all the node" is referred to as inference. PGMs feature prominently in problems in areas such as computer vision, speech recognition, communication, and bioinformatics. However, most often these graphs do not have a matrixlike structure and the inference on the them is often performed via iterative methods, such as belief propagation (Pearl 1988) or Monte Carlo sampling (Mackay 1998). Furthermore, while there has been successful deployment of machine-learning algorithms for perceptual applications such as computer vision and listening on the cloud, there are as yet limited examples of mobile solutions. Our work in this article is precisely at this intersection: We seek to understand if PGM inference formulations-which are usually of an iterative character-can be made "hardware friendly" for the especially challenging domain of resource limited (gates, memory, latency, power, etc.) mobile computing platforms.

To concretize this area of interest, we focus on one such perceptual ML application: mobile sound source separation. Sound source separation can be used to separate human voice from background noise. In the real world, humans listen to a mixture of multiple sound sources and the brain separates out the sources naturally, which is referred to as the cocktail party problem (Cherry 1953). However, performing such separation remains difficult for machines. While there have been serious advances in source separation via ML algorithms, there are still fundamental issues such as the tradeoff between usable quality and computation complexity. Our proposed plan to implement source separation in a practical mobile form raises additional challenges-notably real-time latency and low-power constraints-because (as we shall demonstrate later) conventional ML algorithms in software take a long time to execute and also consume considerable power when hosted on a conventional mobile processor.

When minimal or no information is provided about the sources or the mixing process, this problem is called blind source separation (BSS) (Cardoso 1998). Assume a use setting where BSS is performed on a mobile phone with two microphones-one aimed at the speaker and one aimed away from the speaker. This is a situation where the number of mixture signals is less than the number of sources. We focus on a recently introduced formulation of BSS that models the problem as an undirected PGM, specifically a Markov random field (MRF), and use maximum a posteriori 
(MAP) inference on the model to find the best separation of sources, given the mixtures captured on the microphones (Kim et al. 2012). The idea is to construct a pairwise MRF where each of the nodes represents a distribution of the desired and the interfering sound sources for a timefrequency point of the spectrograms obtained by taking the short-time Fourier transform (STFT) of the sound mixtures from the microphones. We then impose a binary labeling problem on our MRF, where each node (or time-frequency point) will be classified as the desired ("0") or the interfering ("1") sound source to find a mask that can be used to mask the sound mixtures to separate the sources and reconstruct it by inverse short-time Fourier transform (ISTFT). This task of finding the best set of labels given the MRF that describes the distribution can be done as MAP inference. MAP inference is most often intractable and approximate methods are commonly used. There are several strategies for MAP inference, such as belief propagation (Pearl 1988) and random sampling (Mackay 1998). Markov chain Monte Carlo (MCMC) random sampling methods are asymptotically exact and nonparametric but computationally more expensive-which is one strong motivator for our interest in hardware acceleration of them. In this work, we use Gibbs sampling, which is a widely used MCMC algorithm for approximate MAP inference. For completeness, we note that Gibbs sampling is not only used for inference in graphical models but also for other ML applications such as restricted Boltzmann machines (RBMs) and even deep belief networks (DBNs) (Hinton et al. 2006). The key idea is to generate samples by sweeping through each variable or node to sample from its conditional distribution with all other variables fixed and repeat until convergence. The simplicity of the algorithm and its good performance on different applications comes with the cost: Its iterative nature and the search for convergence means longer runtime.

To crystallize our focus on the resource requirements to implement an iterative inference methods on PGMs, we consider a use case where source separation is deployed on a mobile phone to separate the desired voice of the speaker from the interfering noise sources around it, e.g., talking over the phone in a loud airport. This use case requires streaming with a low real-time latency while consuming low power. Failure to meet any of these requirements will make it impractical for real-world usage. For example, the International Telecommunication Union (ITU) describes the recommended mouth-to-ear delay, the acceptable delay from the speaker's mouth to the listener's ears, to be less than $200 \mathrm{~ms}$ (ITU 2003). Furthermore, a typical latency of mouth-to-ear delay of an LTE network is assumed to be approximately 160ms (Holma and Toskala 2011), which means a practical source separation implementation should take less than $40 \mathrm{~ms}$. It is impractical to use source separation while talking on the phone if it drains the battery fast due to complex computations. This work describes our custom hardware implementation of sound source separation on a working FPGA and a simulated virtual ASIC design to satisfy these real-world constraints.

There are prior studies that focus solely on accelerating Gibbs sampling inference for Markov Random Fields, which we have used for source separation. One approach is to accelerate through incorporation of novel devices (Das et al. 2015; Mansinghka et al. 2008; Wang et al. 2016). While we are not the first to implement hardware-based Gibbs sampling inference, we are the first to implement using standard digital CMOS-based fabrics (FPGA, ASIC), which is rather more amenable to practical usage. Furthermore, our implementation is the first real-time streaming hardware implementation of source separation using MAP inference on an MRF (Kim et al. 2012; Ko and Rutenbar 2017). There has been some prior work on hardware implementation of source separation using independent component analysis (ICA) (Hyvärinen et al. 2004), which inverts the mixing process. ICA requires the setup to be an overdetermined case, which requires as many microphones as the number of sources; but in practice, we often deal with a more complex underdetermined mixing problem with fewer microphones than sources, which works better with our MRF-based approach (Kim et al. 2012). Additionally, our preferred MRF model has other useful advantages, such as the 
ability to incorporate not only likelihood but also prior information such as the smoothness of the sound mixture spectrograms, which allows more robust separation. We are also able to use expectation-maximization (EM) in the hardware itself for online update of the MRF parameters which is useful with moving sound sources as well. In addition, previously reported ICA-based implementations (Charoensak and Sattar 2005; Kim et al. 2003; Shyu et al. 2008) fail to meet latency, power, or quality requirements for a practical sound source separation on a mobile phone.

The rest of this article is organized as follows: Section 2 briefly introduces how the source separation problem is modeled as inference on an MRF. Section 3 outlines MRF MAP inference, via the MCMC method of Gibbs sampling, and how we update essential MRF parameters in an online manner using EM. Section 4 describes our MRF source separation hardware architecture, along with details on how each stage of the overall hardware pipeline is designed to achieve real-time streaming with low latency. Section 5 provides additional details of core optimizations we need to allow our design to meet speed constraints, along with a discussion of key tradeoffs, such as the size of the MRF and the number of sampling inference iterations to find an acceptable MAP labeling. Section 6 summarizes our experimental setup, our benchmarks, and our software reference models and presents two sets of hardware implementation results: a working, streaming, real-time FPGA design and a simulated $45 \mathrm{~nm}$ virtual ASIC design. A discussion on related work is also included. Section 7 offers concluding remarks.

\section{SOURCE SEPARATION USING MARKOV RANDOM FIELD}

A widely used BSS approach to solve an underdetermined source separation problem is timefrequency masking (Roman et al. 2003; Yilmaz and Rickard 2004). Binary masking methods search time-frequency binary masking values that denotes whether each point in the spectrogram belongs to the desired or the interfering source under an assumption that the spectrograms for each source are sparse and do not have significant overlap in energy for each time-frequency points. Our proposed implementation formulates an MRF describing the probabilistic relationship between the set of unknown binary labels $x$ for all of the nodes corresponding to each time-frequency point given the sound mixtures information $y$ captured from the microphones. To represent the sound mixtures information $y$, we use an auditory cue commonly used for sound localization called interaural level difference (ILD) (Birchfield and Gangishetty 2005). ILD can be found as the log-ratio of the energy values seen at each of the time-frequency point, between the spectrograms of the two microphones as shown by

$$
\mathcal{A}_{i}=20 \log \frac{Y_{i}^{L}}{Y_{i}^{R}},
$$

where $Y_{i}^{L}$ and $Y_{i}^{R}$ correspond to the energy of time-frequency point $i$ of each of the spectrograms. This probabilistic relationship can be described as the problem of computing a conditional probability distribution, or a posterior distribution, $P(x \mid y)$, over the unknown variables $x$ given observed variables $y$. By Bayes' Rule, the posterior distribution is given by

$$
P(x \mid y)=\frac{P(y \mid x) P(x)}{P(y)},
$$

where $P(y \mid x)$ and $P(x)$ are likelihood and prior distributions, whose product is the equivalent to the joint distribution $P(x, y) . P(y)$ can be thought of as a normalizing constant that can be found by marginalizing the joint distribution by $x$. The probabilistic relationship between the binary labels $x$ and the ILDs $y$ can be described as the likelihood distribution while the relationship between the binary labels $x$ is represented as the prior distribution. 


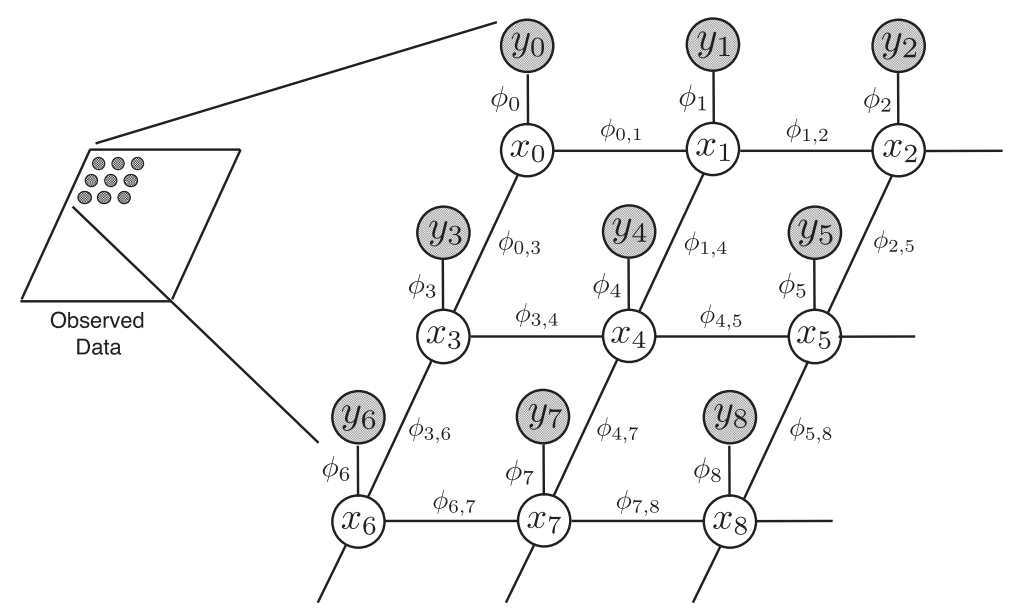

Fig. 1. A partial representation of pairwise MRF that describes the relationship between nine observed variables $y_{i}$ (shaded nodes) from the input data and nine hidden variables $x_{i}$ (white nodes) is shown. The likelihood distribution is represented by edges between each $x_{i}$ and $y_{i}$ pair while the prior distribution is described by edges between each $x_{i}$ and its neighboring nodes.

Source separation MRF is formed by assigning a binary mask label $x \in\{0,1\}$ to each timefrequency point of the spectrograms obtained by taking the STFT of the sound mixtures captured from the microphones. Figure 1 shows a partial representation of the MRF constructed, where a set of all nodes and a set of all edges between nodes in the entire MRF $\mathcal{G}$ are given by $v$ and $\varepsilon$, respectively. Given a node $i \in v$, the relationship between the label on the node $x_{i}$ and the observed ILD $y_{i}$ is represented as a factor $\phi_{i}$. The edge between node $i$ and $j,(i, j) \in \varepsilon$ represents the distribution between the neighboring nodes using a factor $\phi_{i, j}$. The posterior distribution of MRF is the product of all node and edge factors given as

$$
P(x \mid y)=\frac{1}{Z} \prod_{i \in v} \phi_{i}\left(x_{i}, y_{i}\right) \prod_{i, j \in \varepsilon} \phi_{i, j}\left(x_{i}, x_{j}\right)
$$

where $Z$ is a normalizing constant that is the sum of all possible values of $\phi$. Given the MRF, we would like to find the maximizing assignment of labels $x$ of Equation (3) by performing maximum a posteriori (MAP) estimation. To replace the multiplications to summations we can represent this distribution in log domain by taking the -log of Equation (3) as the energy function,

$$
E(x, y)=\sum_{i \in v} \theta_{i}\left(x_{i}, y_{i}\right)+\sum_{i, j \in \mathcal{E}} \theta_{i, j}\left(x_{i}, x_{j}\right)
$$

where $\theta_{i}\left(x_{i}, y_{i}\right)$ and $\theta_{i, j}\left(x_{i}, x_{j}\right)$ are called data cost and smoothness cost, respectively (Scharstein and Szeliski 2002; Szeliski et al. 2008). Given Equation (4), the MAP problem can be formulated as an energy minimization problem on $\mathcal{G}=(v, \varepsilon)$, where now the objective is to find the assignment of labels that will minimize the costs or the energy function:

$$
x^{*}=\arg \min _{x} P(x \mid y)=\arg \min _{x} E(x \mid \theta)=\arg \min _{x}\left\{\sum_{i \in v} \theta_{i}\left(x_{i}\right)+\sum_{i, j \in \varepsilon} \theta_{i, j}\left(x_{i}, x_{j}\right)\right\} .
$$




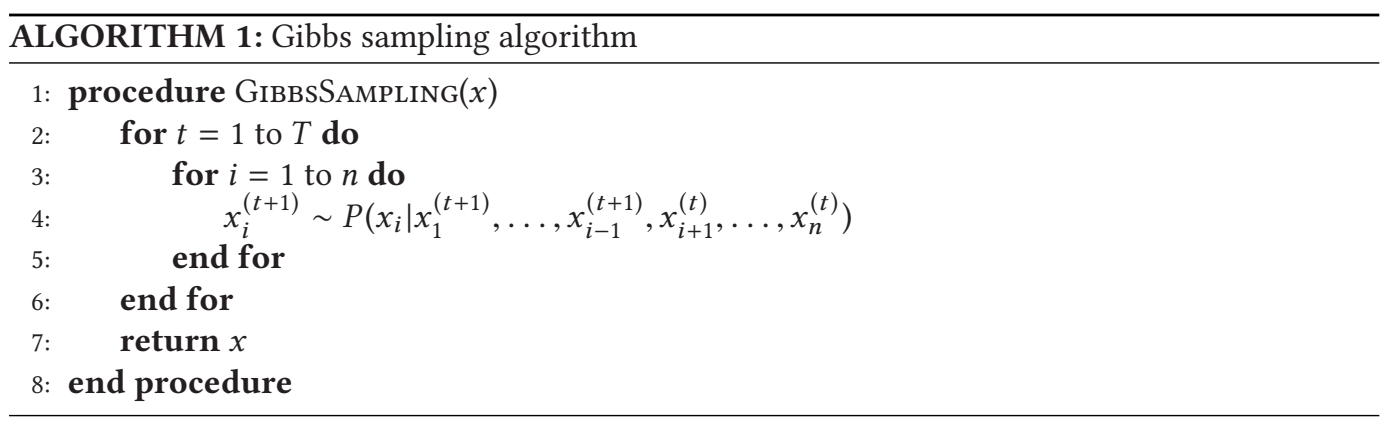

We follow the Gaussian-based data cost formulation of our previous work (Kim et al. 2012) in which the data cost is defined as a function of two Gaussians,

$$
\theta_{i}\left(x_{i}\right)=\left\{\begin{array}{ll}
\frac{\left(A_{i}-\mu_{0}\right)^{2}}{2 \sigma_{0}^{2}} & \text { if } x_{i}=0 \\
\frac{\left(A_{i}-\mu_{1}\right)^{2}}{2 \sigma_{1}^{2}} & \text { if } x_{i}=1
\end{array},\right.
$$

where $A_{i}$ is the ILD at node $i$, given by Equation (1). The means, $\mu_{0}$ and $\mu_{1}$, and the variances, $\sigma_{0}$ and $\sigma_{1}$, correspond to the mean and variance of each sound component of the energy ratio Gaussians. The smoothness cost $\theta_{i, j}\left(x_{i}, x_{j}\right)$ models the prior preference of two neighboring nodes, defined on edge $(i, j) \in \varepsilon$, to encode spatial locality in frequency and time domain in the spectrogram as follows (Kim et al. 2012):

$$
\theta_{i, j}\left(x_{i}, x_{j}\right)=\left\|x_{i}-x_{j}\right\|^{2} .
$$

The goal of the optimization problem (5) is to find the set of labels $x$ among all possible per-node label choices that minimize the objective function.

\section{GIBBS SAMPLING INFERENCE AND PARAMETER ESTIMATION}

In recent years, several algorithms have been proposed to solve the above MRF-MAP inference problem (Boykov and Jolly 2001; Kappes et al. 2013; Kolmogorov 2006; Scharstein and Szeliski 2002; Szeliski et al. 2008; Wang et al. 2013). While many of these algorithms are successful in solving the problem, it is still intractable to run these algorithms in real time for common perceptual applications like sound source separation or computer vision applications. One large class of algorithms used for the inference problem in MRFs are Markov chain Monte Carlo or sampling-based methods. Advantages of these algorithms include simplicity of implementation and theoretical guarantees of converge, while they can be slow to converge, and it can be difficult to diagnose the convergence. We use one of the most widely used MCMC method called Gibbs sampling, which was first introduced in the context of solving MRF for compute vision application (Geman and Geman 1984). Gibbs sampling operates iteratively by choosing a variable in each time step and updating it by sampling from its conditional distribution given the other variables in the model as shown in Algorithm 1. It is especially effective on MRF, because it only needs to observe neighboring nodes that are directly connected the sampled node, the Markov blanket, due to local Markov property, that imposes conditional independence (Koller and Friedman 2009). This allows local computations, since processing a single node requires data accesses only for the neighboring nodes and is attractive on hardware. Furthermore, there are many variants of Gibbs sampling that allow more parallelism with tradeoffs.

In the above formulation of data costs for source separation MRF, the Gaussian parameters for the data cost function are hidden parameters. Without having proper parameters that closely 


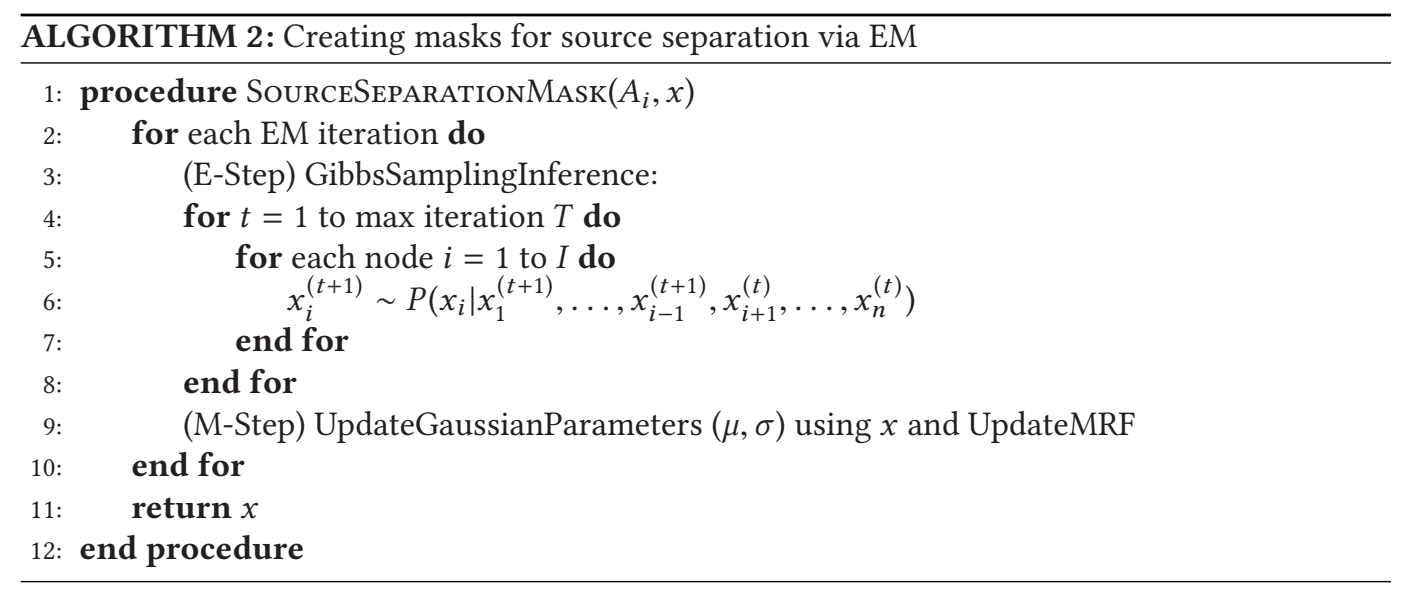

describe the actual distribution, the inference results would not be of high quality. To find the hidden parameters, we can train the MRF offline to learn the parameters by using a labeled dataset. In fact, that is how most mobile deep neural network implementations are done; training is not done on mobile but offline in advance. However, if the parameters are fixed, then source separation only performs well given the specific configuration of sound sources relative to the microphones that corresponds to the energy ratio distribution given by the fixed parameters. This is not very practical for real-world application of source separation where sources could be moving relative to the microphones on the mobile phone. Furthermore, user intervention to modify the parameters are hard to impose on a real-time system.

Just as it is common for image segmentation applications to receive initial seed or hints about the segmentation from the users (Boykov and Jolly 2001), we can also provide initial observations or guesses about the underlying distribution. We can then perform the Expectation-Maximization (EM) (Dellaert 2002; Dempster et al. 1977; Minka 1998; Neal and Hinton 1998), an iterative optimization method, to learn the Gaussian parameters of the distribution on the fly while performing source separation. Each iteration of EM consists of an Expectation (E) step, which finds the distribution for the unobserved variables, given the observed variables and the current estimate of the hidden parameters, and a Maximization (M) step, which re-estimates the parameters using the distribution found in the E-step.

For source separation, we start with rough guesses of the Gaussian parameters for the energyratio distribution for the data cost function of MRF, based on the geometric orientation of the microphones and the sources. We assume that the desired sound source is closer to the microphone. When we perform the EM algorithm, E-step finds the distribution of labels given the estimate of the Gaussian parameters by performing Gibbs sampling inference on the MRF, and M-step updates the parameters to a more accurate estimation using the distribution of labels found as described in Algorithm 2. By performing M-step the data cost is updated and E-step will be performed on the updated MRF resulting from it. The EM iterations are repeated until convergence and the inference results on the final MRF represents the MAP solution to the source separation problem in the form of a binary mask that can be used to separate the sources. This is especially useful in scenarios where the sources are not stationary with respect to the microphones.

\section{REAL-TIME STREAMING MARKOV RANDOM FIELD SOURCE SEPARATION}

In this section, we present a real-time (low latency) streaming implementation of the proposed MRF source separation. The high-level view of the implementation, from input to the output sound 


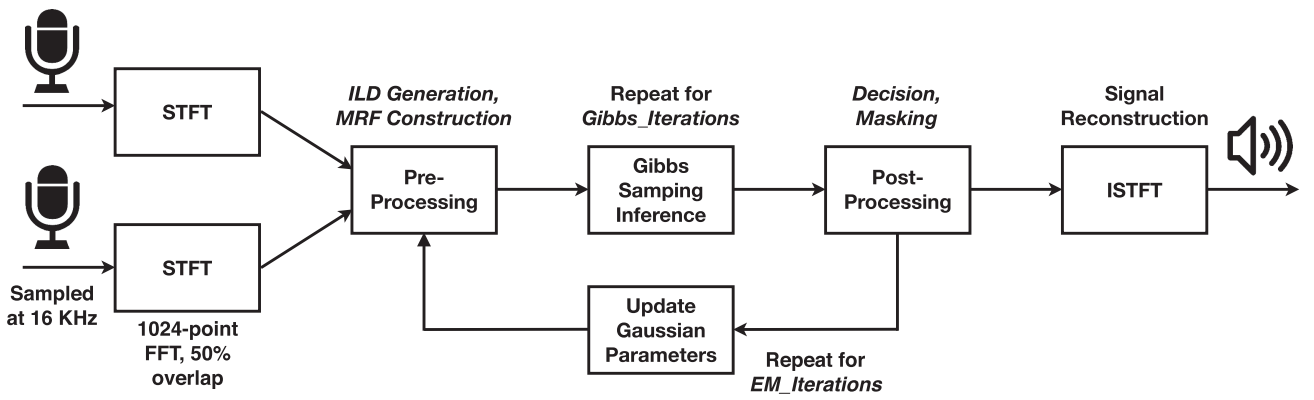

Fig. 2. The input sound mixtures are streamed in, processed, and reconstructed as separated sound streams.

mixtures, is shown in Figure 2, with each corresponding stages of a streaming system. We will discuss each of stages in detail, providing explanation for design choices made with the goal of achieving a real-time and low-power streaming source separation on a mobile phone. The proposed implementation was designed following these real-world design constraints:

- The design should be able to handle constant stream of audio input. To have any practical use such as suppressing noise while talking on the phone in a noisy environment, source separation should be implemented in a streaming fashion.

- The design should maintain the mouth-to-ear latency below 40ms. International Telecommunication Union's recommendation (ITU 2003) regarding mouth-to-ear delay latency indicates that most users are "very satisfied" as long as latency does not exceed 200ms. However, a typical latency or mouth-to-ear delay of LTE network is assumed to be approximately $160 \mathrm{~ms}$ (Holma and Toskala 2011), which means latency should be smaller than $40 \mathrm{~ms}$ to achieve "very satisfied" quality of service.

- The design should not consume more than a watt and hopefully less than $100 \mathrm{~mW}$. Using source separation in regular voice talk should permit several hours of usage on a typical mobile phone.

\subsection{Spectrogram Generation}

In the first step, the pair of audio input streams are converted to a pair of spectrograms by performing STFT. We assumed that the input signals are sampled at $16,000 \mathrm{~Hz}$, which is highest of the sampling rate supported by a typical mobile phone. The higher sampling rate results in more temporal resolution that can be useful for achieving better separation of the mixtures. Furthermore, size of the FFT determines the frequency resolution of the resulting spectrogram. We have decided to use 1,024-point FFT with 50\% overlap and a cosine window. This results in a single input sound frame of size 1,024 samples and one FFT on a single frame will correspond to a single column on the constructed MRF with 513 rows, which is the by-product of the FFT size. The sampling rate, FFT size, and the amount of overlap determines the temporal resolution of the resulting MRF.

\subsection{ILD Generation}

The ILD matrix used for computing data cost function of MRF, is generated by taking the log ratio of energy corresponding to each of the time-frequency points in the spectrogram given by Equation (1). Figure 3 shows the ILD generation architecture for a single time-frequency point in the spectrogram. STFT results stored in intermediate buffers are streamed into the ILD generation block that is pipelined with each stage that consists of Q16.16 fixed-point elementary arithmetic units. The log function used to calculate ILD is implemented via $\log _{2}$ block in Q16.16 fixed-point 


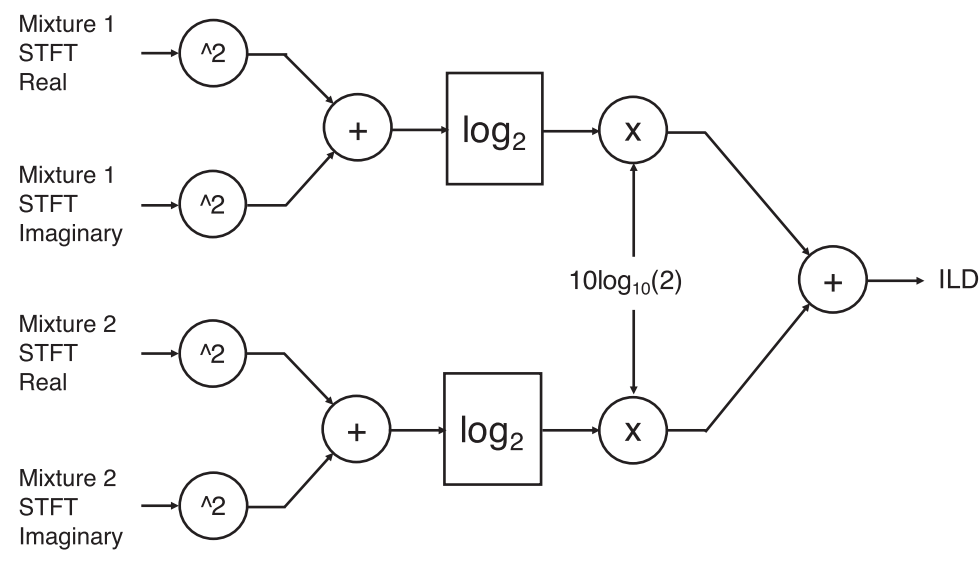

Fig. 3. Interaural level difference generation block receives Q20 values as inputs and processes them in Q16.

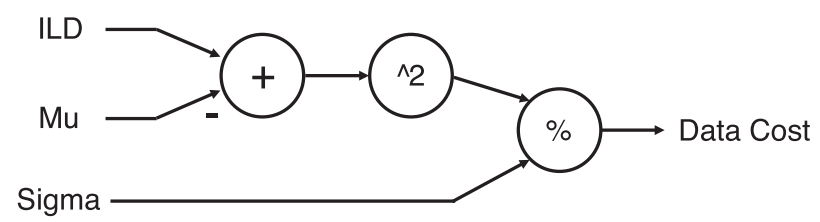

Fig. 4. Data cost generation block uses Q16 precision for all the arithmetics. The ILD values are streamed in from ILD generation block and the Gaussian parameters are from the previous EM iteration.

based on methods that use a lookup table-based Taylor approximation (Mitchell 1962; Gutierrez and Valls 2011) to minimize resources and latency. The lookup table consist of 256 16-bit elements. A constant of $10 \log _{10}(2)$ is multiplied with result of $\log _{2}$ block to produce $\log _{10}$ results. When a single frame is processed, it will result in a single column of a spectrogram and the ILD matrix.

\subsection{MRF Construction}

The construction of MRF requires data cost and smoothness cost computation. The data cost generation block is shown in Figure 4. Every cycle, it takes an ILD value from a single time-frequency point in spectrogram along with current Gaussian parameters to calculate a new data cost for it. While the figure looks very simple, it is a relatively large block with a divider. Two identical data cost generation blocks are used for each of the binary label values. The registers between each stages were omitted in the figure. Instead of calculating smoothness cost on MRF construction, we use a technique to calculate smoothness costs on the fly when performing Gibbs sampling as shown in Figure 4.

Initially, Gaussian parameters for calculating the data cost of the MRF are approximated based on the location and the orientation of the sources relative to a pair of microphones on a mobile phone. These are updated using EM where E-step is the Gibbs sampling inference and the M-step is updating the Gaussian parameters from the resulting labels from the E-step and modifying the data cost of the MRF.

\subsection{Gibbs Sampling Inference}

For the E-step of the EM parameter estimation, we perform Gibbs sampling on MRF constructed with current Gaussian parameters. Once the data cost values are generated from the corresponding 


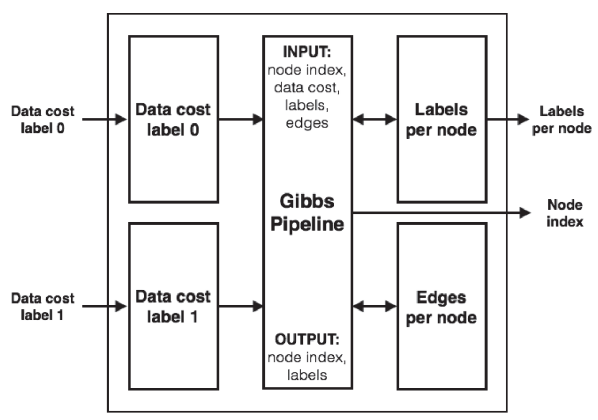

(a)

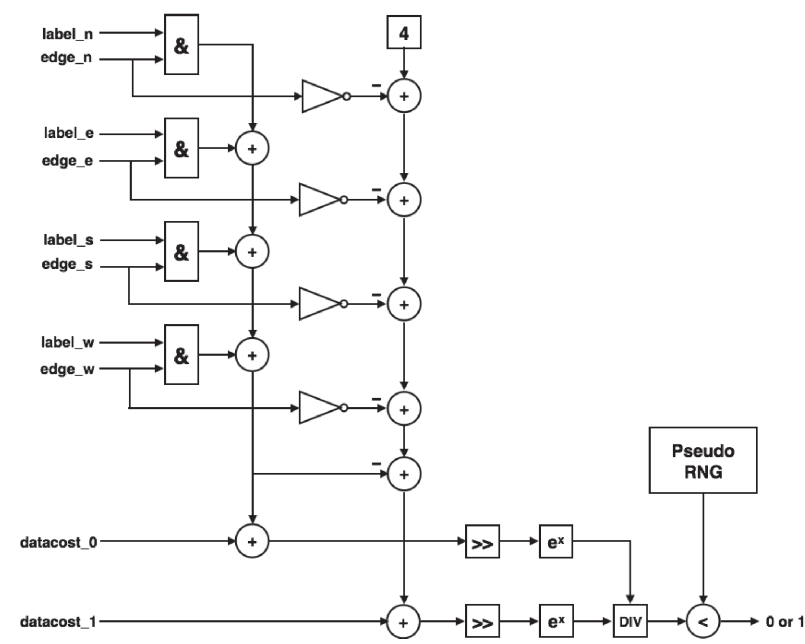

(b)

Fig. 5. The architecture of the Gibbs sampling inference block and the Gibbs sampler are shown. (a) Gibbs sampling inference block caches local copy of all the variables of MRF within the block. The Gibbs pipeline creates a list of all nodes to be sampled along with indices, data costs, labels, and edges per node. (b) Gibbs sampler receives labels of the neighboring pixels and also the existence of edges to its four neighbors.

input frame, it is passed into Gibbs sampling inference block, which locally caches the data costs and the label assignment for all the nodes in the entire MRF. The size of the local memories for storing the data costs and the label assignments are determined by the size of the MRF.

Figure 5 shows the architecture of the Gibbs sampling inference block and the Gibbs sampler, which calculates smoothness on the fly and samples one node given the data cost and labels of the neighboring nodes. Gibbs sampling pipeline is designed to take up to 1,024 nodes at a time and process them sequentially. The input to the pipeline is a series of nodes with corresponding node index, label, labels for the neighboring nodes, and data costs. Instead of being computed in advance along with the data cost, the smoothness cost is computed on the fly using logic shown in Figure 5(b), which takes advantage of the two-dimensional (2D) grid structure of our MRF where each node only depends on four neighboring nodes. The output is the updated labels for each of the nodes with a corresponding node index. Depending on the variant of Gibbs sampling used, the number of Gibbs sampler within the pipeline could be increased to allow multiple chains or support different parallelization schemes. For our implementation, a single processing element 


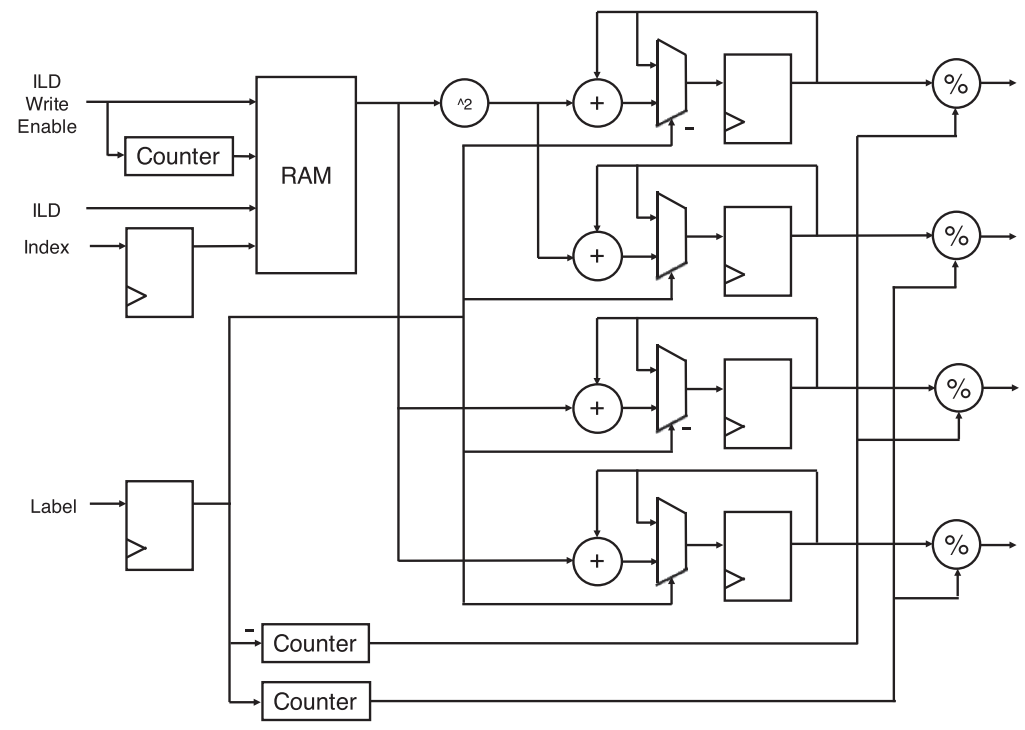

Fig. 6. Gaussian parameters update block takes cached ILD values along with labels corresponding to same node of the graph to update Gaussian parameters based on Gibbs sampling inference results.

provided enough performance while minimizing the latency and power consumption. Once the Gibbs sampling pipeline goes through all columns, it is considered to have completed an iteration of Gibbs sampling.

The exponential function in the Gibbs sampler is implemented in Q16.16 fixed-point, which is a close modification of a method that uses a table-based Taylor approximation (Detrey and de Dinechin 2007), with the 256 element LUT. The pseudo random number generator in the Gibbs sampler is implemented in 32-bit fixed-point using 43-bit linear feedback shift register (LFSR) and 37-bit cellular automata shift register (CASR) (Tkacik 2003). Both of the functions were implemented after precision analysis using a $\mathrm{C}++$ software reference for source separation.

\subsection{Masking, Updating Gaussians, and Output Reconstruction}

Once the Gibbs sampling inference is completed, resulting labels for the MRF is passed onto Gaussian update block to find new Gaussian parameters. This step corresponds to the M-step in EM algorithm. The updated Gaussian parameters are used to modify data costs for the MRF, and the next iteration is performed, and so forth. Once the number of EM iterations is reached, the labels are passed to the masking block, where the FFT results for the current frame are masked and passed onto the ISTFT block. The ISTFT block maintains the previous frame data, which can be used with current frame data to perform IFFT and reconstruct the output separated sources.

Figure 6 shows the architecture of the Gaussian update block. As shown, ILD value along with binary labels and corresponding indices are used to generate new mean and variance. It is an iterative pipelined architecture with four dividers. Once all the ILD values and binary values for the MRF are iterated, new values will be passed onto the data cost generation block to update the MRF. This completes the EM iteration.

\section{ARCHITECTURE OPTIMIZATION}

Figure 7 is the top-level architecture of our proposed streaming hardware. One key feature to note is that the data flow from the left (input) side of the diagram to the right (output) side, which 


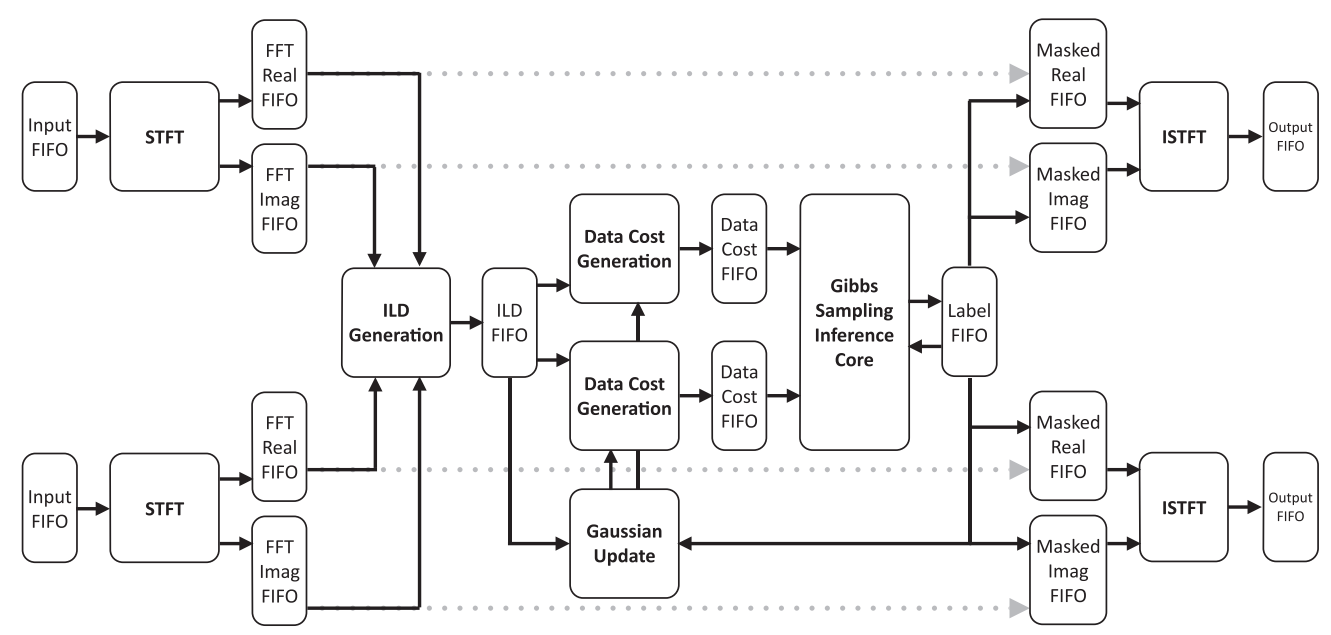

Fig. 7. The proposed source separation architecture using EM and Gibbs sampling is shown. The dotted gray lines show that the STFT results of the input mixtures must be kept until the inference is done to mask and reconstruction it using ISTFT.

follows the dataflow computing idea (Culler 1986; Dennis and Misunas 1975; Gurd et al. 1985). This allows minimum latency from the input to output while taking full advantage of pipelined blocks for maximum throughput as well. The arrows indicate the direction of the data movement. There are two iterative loops in this architecture: One is the Gibbs sampling loop, which is within the Gibbs sampling inference block, and the other is shown by arrows traveling left from label FIFO into Gaussian update block, which realizes the EM loop for updating Gaussian parameters. The use of iterative loops requires numerous buffers or FIFOs as shown. One of the key variables for overall performance, in terms of latency and throughput, power consumption and size (or cost) of the hardware is size of the MRF.

\subsection{Constructing Optimal MRF: Data Cost and Label Reuse}

To take advantage of the spatial locality of the sources in both the frequency and time domain, a larger MRF is preferred to allow more prior information to be included. Note that a single column of the MRF does not contain much information about the approximated Gaussian mixtures. Although it will have a smoothness prior in the vertical (frequency) direction, it will not have the horizontal (temporal) prior information. To circumvent this problem, we construct an MRF not only using the current frame of audio being processed but also several previous frames to take advantage of smoothness in the temporal direction.

The larger the MRF is, the better the separation of sources will be. However, a larger MRF means more memory, computation, and power consumption. We must determine the optimal size of the MRF while considering quality of the separation, memory, latency, an power tradeoffs.

We first focus on latency of the proposed architecture. To estimate expected latency, we look at how long Gibbs sampling inference would take for a given architectural setup. If we denote one Gibbs sampling operation as the atomic operation, then the total number of Gibbs sampling operations (GSops) for estimation and inference is given by

$$
\text { GSops }=513 *(\text { \#of Frames }) *(\# \text { of GibbsSamplingIterations }) *(\# \text { of EMIterations }),
$$


Input sound stream
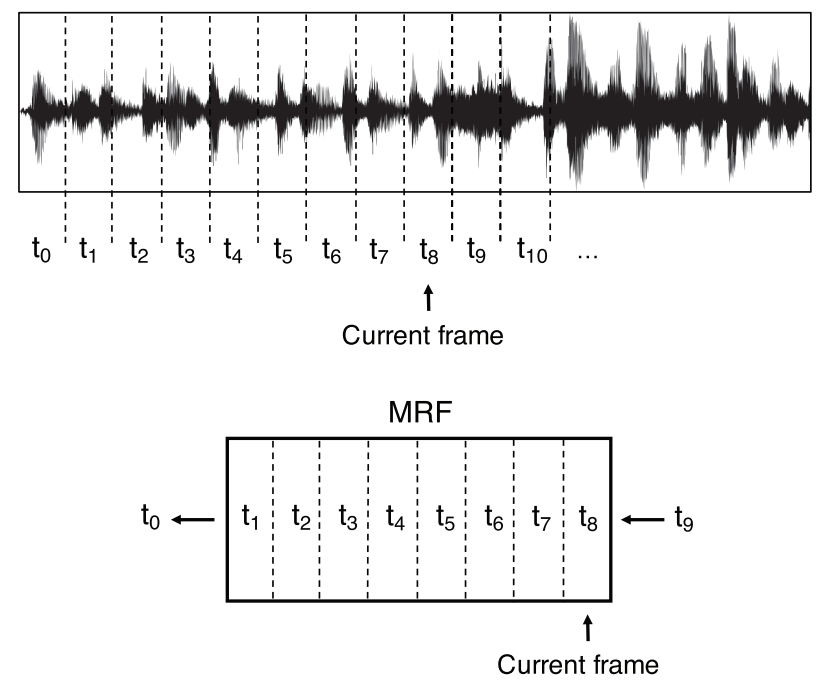

Fig. 8. The constructed MRF contains information from eight input sound frames: one current and seven previous frames. When a new sound frame enters the system, the column of MRF corresponding to the oldest frame in the MRF is retired and new frame fills the void. This enables the streaming implementation with minimal hardware overhead while taking advantage of the temporal information from previous frames for faster convergence and better accuracy.

which increases linearly with the number of frames used to construct MRF. Furthermore, the size of the buffers required to hold MRF variables will be proportional to the number of frames per MRF. The local memories to store intermediate data for two iterative loops, such as data cost buffers for each label values and label buffers, are the largest memories in the design, determined by the size of the MRF.

To optimize the size of the MRF, instead of constructing a new MRF from scratch, we take advantage of the streaming architecture by appending the existing MRF with the new input sound frame information while retiring the part of the MRF that corresponds to the oldest sound frame as shown in Figure 8. On top of this, we reuse the labels determined from previous inference on the MRF to further reduce the total size of the MRF. Instead of starting from scratch for each sound frame, we take the label values from the previous inference run as the starting point for the Gibbs inference process. This allows faster convergence and as a result, a huge reduction of latency and memory. This "warm start" of Gibbs inference by keeping older frames and labels from the inference of the previous input frame allows us to use significantly smaller MRF with a very small number of iterations for convergence.

To determine a practical size for a hardware implementation, a set of experiments were made with varying number of frames, Gibbs sampling iterations, and EM iterations. First, to find the appropriate number of frames, or number of columns in MRF, we must verify that source separation's quality is acceptable for a given size. The qualitative performance of source separation can be measured by Signal-to-Distortion Ratio (SDR), which is widely used as an objective separation quality evaluation metric (Vincent et al. 2006). SDR is found by comparing the original spectrogram with the masked spectrograms corresponding to the separated signals. With the number of EM and Gibbs sampling iterations set to a high number to prevent underfitting, we vary the number of frames and measure SDR. The results plotted in Figure 9 show that source separation does 


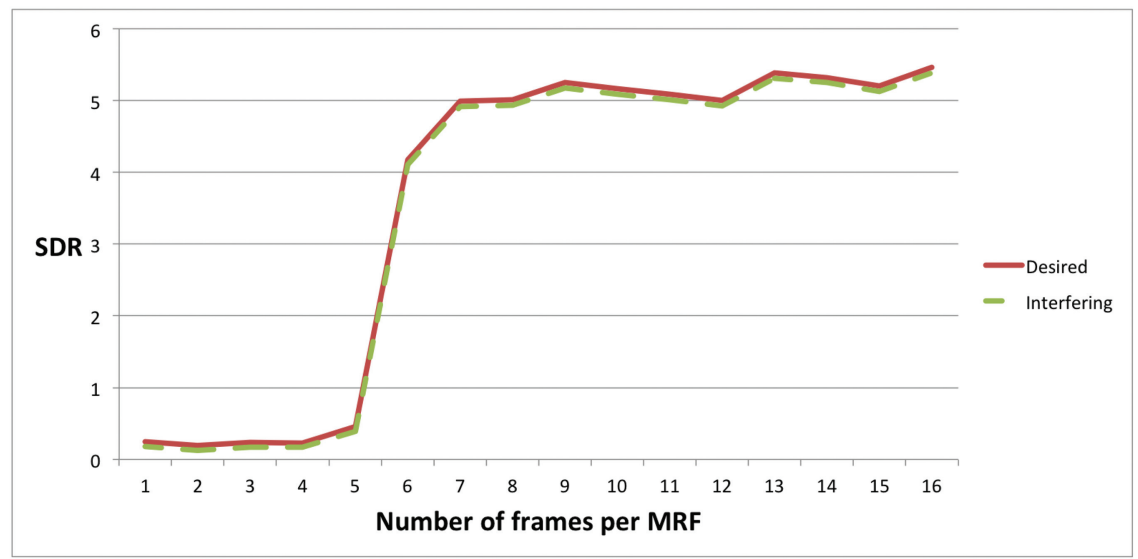

Fig. 9. SDR starts improving with at least six columns (frames) of MRF.

not work that well with five frames or fewer and gives usable quality $(5 \mathrm{~dB})$ or higher for seven frames and more. For our implementation, we decided to use eight frames to minimize memory size and also to reduce the runtime while achieving good SDR.

Two additional experiments were performed while fixing the MRF size to eight frames. If the Gibbs sampling iterations were set to a high number to avoid not converging, then even one iteration of EM showed results that are $5 \mathrm{~dB}$ or higher. However, if the EM iterations were set to a high number while sweeping through Gibbs sampling iterations, then we observed that for three or fewer iterations, SDR values were poor. While using a high number of Gibbs sampling iterations resulted in good SDR for even one iteration of EM, it may not extend to situations where the distribution is changing rapidly or if the sound sources are moving too fast. Without having some EM iterations to account for these shift in Gaussian parameters, the quality of the separation will be compromised. Based on these observations, we have determined the number of EM and Gibbs sampling iterations to four each.

\subsection{Mixed Precision Architecture}

Our system uses a 32-bit fixed-point arithmetic with 16 fractional bits (Q16.16) for most parts of the design. The number of fraction bits required to convert the floating-point arithmetic of the source separation algorithm was determined by checking dynamic range and an adequate amount of precision required to produce correct values within the system. The number of fractional bits is increased in certain parts of the design, such as the Gibbs sampling inference core, where we deal with probabilities and very small numbers. We extend the fractional bits to nearly all 32 bits to provide more precision. Another exception to this fixed-point number format is input and output stream values that are fractions with magnitude is less than 1 . To maximize precision, input to STFT and ISTFT to output uses 31 fractional bits (Q31), with a sign bit.

\section{EXPERIMENTS AND RESULTS}

We have several different implementations of the algorithm, each designed to evaluate a different aspect of the design. This section provides description for experimental audio setup, software reference using $\mathrm{C}++$ benchmarked on both desktop and simulated mobile phone processors, FPGA hardware implementation for functional correctness and the ability to run "fast enough," and virtual ASIC design synthesized in standard 45nm process for estimating clock frequency and power. 


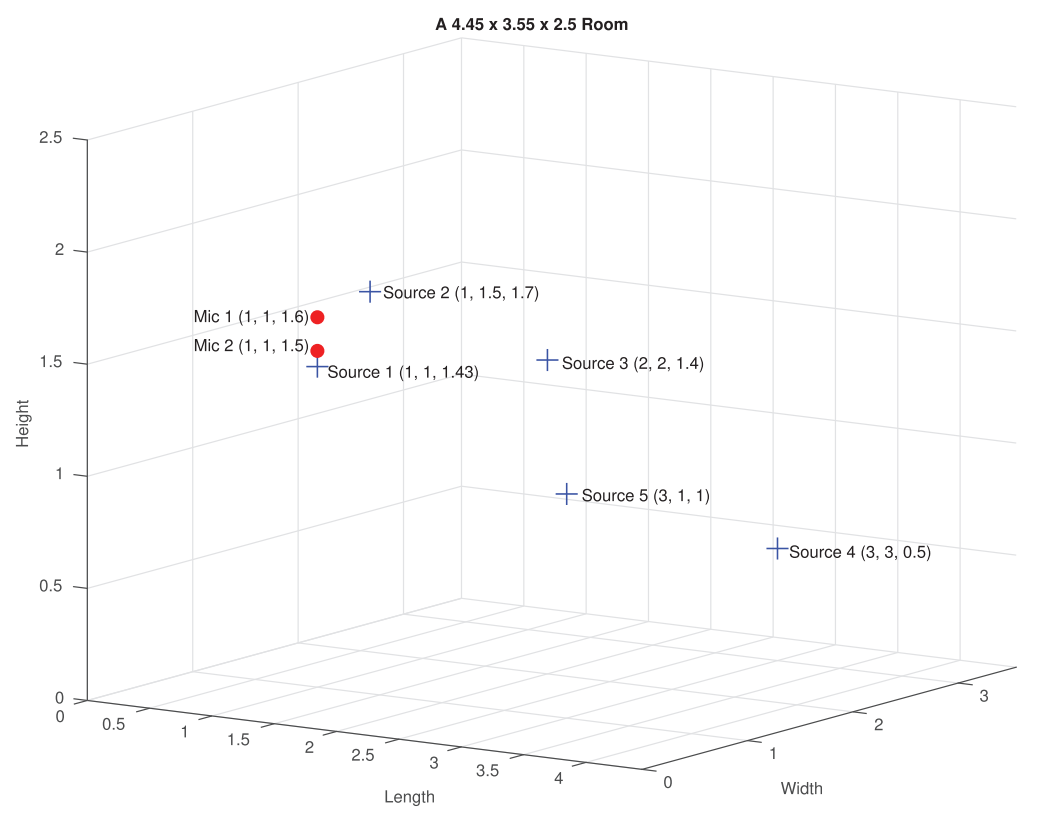

Fig. 10. A $4.45 \times 3.55 \times 2.5$ room with a mobile phone and five sound sources is used. Source 1 is the desired speech signal, Source 2 is the interfering speech signal, Source 3 is a blender, Source 4 is a rolling can, and Source 5 is a washer.

\subsection{Experimental Audio Setup}

To evaluate our implementation, we chose two speech signals, one female and one male, from the TIMIT corpus (Garofolo et al. 1993) as the input sound sources. These speech signals were sampled at $16,000 \mathrm{~Hz}$ and encoded using 16-bit PCM. We modeled the audio input as a pair of microphones separated by $15 \mathrm{~cm}$, a value derived from the length of a typical mobile phone. We created two convolutive mixtures with simulated room reverberations corresponding to each microphone by using Roomsim Matlab toolbox (Campbell et al. 2005). A comprehensive mixture of a desired speech signal, another speech signal, a blender, a rolling can, a washer, and additional microphone noise in the mobile phone environment from Kim et al. (2012) is used for the evaluation. The dimensions of the room and the location of the sound sources and the microphones on the cellphone are shown in Figure 10 where the units are in meters.

\subsection{Software Reference Architecture}

We have implemented the software reference of MRF source separation using $\mathrm{C}++$ and benchmarked it on Intel Xeon X6550 2GHz. The high-performance CPU took $31.966 \mathrm{~ms}$ to run sound source separation on $32 \mathrm{~ms}$ of input audio, which is the smallest granularity given $16 \mathrm{KHz}$ sampling rate and 1,024-point STFT with 50\% overlap. This essentially means that at least $64 \mathrm{~ms}$ of latency will be consumed for the C++ implementation on Xeon. ITU's "very satisfied" criterion for mouthto-ear delay is $200 \mathrm{~ms}$ or below. As stated previously, LTE network's delay is approximately $160 \mathrm{~ms}$ (Holma and Toskala 2011), and the $64 \mathrm{~ms}$ latency on the Xeon is too high to achieve the quality of service recommended by ITU. To estimate the performance of our software reference on a mobile processor, we ran our code on a cycle-accurate simulator GEM5 (Binkert et al. 2011) with an ARM Cortex-A9 model. It took 23.32 simulation seconds to run a 4-second batch of audio. Using the the 
Table 1. FPGA Resource Utilization

\begin{tabular}{|l|c|}
\hline Resource & Utilization \\
\hline Slice Register & $101302 / 207360(48 \%)$ \\
\hline Slice LUT & $90280 / 207360(43 \%)$ \\
\hline Slice LUT FF & $119300 / 207360(57 \%)$ \\
\hline BRAM & $113 / 288(39 \%)$ \\
\hline DSP & $36 / 192(18 \%)$ \\
\hline
\end{tabular}

Table 2. Memory Instances

\begin{tabular}{|c|c|}
\hline Memory Size & Number of Instances \\
\hline $512 \times 32$ & 8 \\
\hline $1,024 \times 8$ & 2 \\
\hline $1,024 \times 16$ & 2 \\
\hline $1,024 \times 32$ & 20 \\
\hline $8,192 \times 4$ & 1 \\
\hline $8,192 \times 32$ & 3 \\
\hline Total Size & $207 \mathrm{~KB}$ \\
\hline
\end{tabular}

CPU statistics produced from GEM, we use McPAT (Li et al. 2009) to estimate the power of our software reference. The peak power consumption on ARM Cortex-A9 is estimated to be $3.661 \mathrm{~W}$, which is unacceptable for prolonged usage on a mobile phone. Assuming that most mobile phones have roughly $2,000 \mathrm{mAh}$ and nominal voltage of $3.8 \mathrm{~V}$, the energy is $7.6 \mathrm{Wh}$. This barely allows the phone to run the application for 2 hours even neglecting other consumptions.

\subsection{FPGA Implementation}

We implemented a fully synthesized Verilog version of our design running on a Convey HC1 hybrid-FPGA platform (Bakos 2010; Brewer 2010). The platform consists of Intel Xeon 5138 $2.13 \mathrm{GHz}$ dual-core host processor, and four Xilinx Virtex 5 (V5LX330) FPGA coprocessors running at $150 \mathrm{MHz}$ each. The system has a single cache-coherent shared virtual memory, which allows easier data transfers and communication between the host and the co-processors. Our design was able to fit on one Virtex 5 with resource utilization shown in Table 1.

Our system uses Q16.16, a 32-bit fixed-point arithmetic with 16 fractional bits (Q16). The number of fraction bits required to convert the floating-point arithmetic of the source separation algorithm was determined by checking dynamic range and adequate amount of precision required to produce correct values within the system. The exception to this fixed-point number format is input and output stream values that are fractions with magnitude less than 1 . To maximize precision, input to STFT and ISTFT to output uses 31 fractional bits (Q31), with a sign bit. The iterative nature of the algorithm requires its intermediate results such as MRF data cost and labels to be stored in buffers resulting in numerous FIFOs as shown in Figure 7. The size and number of memory instances have been listed in Table 2, with the total size of $207 \mathrm{~KB}$. The hardware is fully parameterized and can be set to allow expansion to other larger inference applications.

For our implementation, the goal was to meet the mouth-to-ear delay recommendation of $200 \mathrm{~ms}$ of the ITU (ITU 2003) as we envisioned its application on a cellphone. With the FPGA running at $150 \mathrm{MHz}$, we were able to achieve $1.601 \mathrm{~ms}$ latency from the input to the output stream while running four iterations of EM and four iterations of Gibbs sampling. This is equivalent to $22 \times$ speed-up compared to the ARM software reference. Even with 160ms LTE network delay, we are 
Table 3. A Comparison and a Breakdown of Power for Different Frequencies and Types of Cells

\begin{tabular}{lllll}
\hline & 12tr-rvt-150m & 12tr-rvt-30m & 9tr-uvt-150m & 9tr-uvt-30m \\
\hline Memory & 404.517 & 79.130 & 403.836 & 78.965 \\
Register & 105.565 & 63.091 & 56.852 & 15.811 \\
Sequential & 0.050 & 0.049 & 0.005 & 0.005 \\
Combinational & 46.487 & 46.349 & 8.640 & 5.097 \\
\hline Total & $556.620 \mathrm{~mW}$ & $188.619 \mathrm{~mW}$ & $469.332 \mathrm{~mW}$ & $99.879 \mathrm{~mW}$ \\
\hline Area & $6,731,304$ & $6,735,203$ & $6,554,452$ & $6,548,631$ \\
\hline
\end{tabular}

Note: 12 tr-rvt-150m is 12 -track RVT cells running at $150 \mathrm{MHz}$. 12 tr-rvt-30m is 12 -track RVT cells running at $30 \mathrm{MHz}$. 9tr-uvt-150m is 9-track UVT cells running at $150 \mathrm{MHz}$. 9tr-uvt-30m is 9-track UVT cells running at $150 \mathrm{MHz}$

able to limit the mouth-to-ear delay under 200ms. The resulting implementation requires 64 bits of memory data width and $1.2 \mathrm{~GB} / \mathrm{s}$ of bandwidth.

\subsection{Virtual ASIC Design Study}

While the FPGA results show that a real-time source separation implementation is feasible on the FPGA, it is definitely not practical for a real appliance due to power restrictions. We implemented several designs using the IBM $45 \mathrm{~nm}$ process to synthesize and place and route our design and find the gate count, area and power estimates of different virtual ASIC implementations.

We started our design by using $150 \mathrm{MHz}$ to match that of the FPGA implementation. As Table 3 shows, the power consumption is relatively high with both 12-track RVT cell and 9-track UVT cell designs consuming more than $400 \mathrm{~mW}$. Because our design had really small latency of $1.6 \mathrm{~ms}$, we can definitely use a much lower operating frequency to reduce power. When the operating frequency is reduced to $30 \mathrm{MHz}$, which still meets the previously defined latency requirements, we see that the power consumption drops below 200mW. For 9-track UVT design, it consumes less than $100 \mathrm{~mW}$, which was one of our original design constraints.

In general, designs using 12-track RVT cells resulted in much higher power numbers. This due to the size of the cell being larger and also the lower threshold voltage. The UVT cells have a higher threshold voltage, which means they are slower but consume less power than RVT cells. While the difference of using 9-track UVT cells over 12-track RVT cells is not that great for designs running at $150 \mathrm{MHz}$, it is very noticeable for designs running at $30 \mathrm{MHz}$, with 9-track UVT design consuming almost half as much power as the 12-track RVT design. Furthermore, we observed that SRAM memory power accounts for more than $50 \%$ of power for all but 12-track RVT design, which is still more than $40 \%$ of the power consumption. For 9-track UVT design, memory consumes almost $80 \%$ of the entire power. One thing to note here is that the SRAM cells were only available in UVT cells, which explains why designs with same operating frequency results in similar memory power consumption. This appears to be a side-effect of our current technology library, which is not aimed at low-power mobile uses. When we resynthesized our design using a lower frequency of $20 \mathrm{MHz}$, we achieved a significantly lower power of $69.977 \mathrm{~mW}$. Our virtual ASIC prototype using 9-track UVT cells, whose layout is shown in Figure 11, resulted in gate count of 9,126,222, area of 6.6 $\mathrm{mm}^{2}$. The overall design is attractively small, under 10 million gates. When running at $150 \mathrm{MHz}$ and $20 \mathrm{MHz}$, the power consumption is $8 \times$ and $52 \times$ better than the ARM reference estimate of $3.7 \mathrm{~W}$.

\subsection{Further Optimizations and Related Work}

While the $45 \mathrm{~nm}$ implementation could achieve a low power of $70 \mathrm{~mW}$, it was evident that most of the power resulted from its use of many SRAMs and using an outdated and non-low-power process 


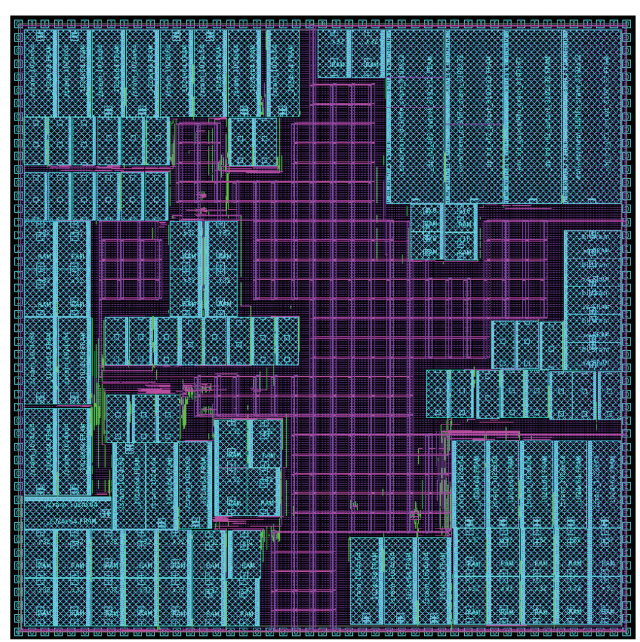

Fig. 11. The ASIC layout using $45 \mathrm{~nm}$ 9-track UVT library is shown. It is evident that our design uses a lot of SRAM, shown by the light blue blocks. The logic cells are distributed in the grid area in the middle.

Table 4. 32nm Memory Instances

\begin{tabular}{|c|c|}
\hline Memory Size & Number of Instances \\
\hline $128 \times 4$ & 33 \\
\hline $128 \times 8$ & 16 \\
\hline $128 \times 16$ & 16 \\
\hline $128 \times 32$ & 291 \\
\hline Total Size & $157 \mathrm{~KB}$ \\
\hline
\end{tabular}

technology. To further optimize power, some architectural modifications were made to our design to allow the buffers holding the MRF variables within the Gibbs sampling inference block to be reduced to a smaller size, without any sacrifices on the inference results. Furthermore, Synopsys $32 / 28 \mathrm{~nm}$ Generic library was used to synthesize our updated design. Both of these modifications hints that our design is still amenable to power optimizations. This resulted in $25 \%$ less memory for the design as shown in Table 4. Note that the list of memory instances are vastly different, because most of the large memories were replaced with smaller memories as the Synopsys $32 \mathrm{~nm}$ library only supported small SRAMs. These two modifications reduced the power of our ASIC implementation down to $40.034 \mathrm{~mW}$.

There has been several different set of previous works related to our MRF source separation using Gibbs sampling inference. There was a hardware acceleration of Gibbs sampling by the use of unconventional substrates that provide some form of entropy. A combinational stochastic circuit called stochastic transition circuit has been proposed (Mansinghka et al. 2008), which provides a way to directly use probabilistic algebra instead of Boolean algebra. However, to use this circuit, an entirely new probabilistic programming interface and a compiler for it, are needed. Das et al. (2015) uses a digital neuromorphic substrate with stochastic leak and threshold properties to implement Gibbs sampling for RBMs and DBNs. It consumes low power but is limited for neuromorphic uses as it is requires the whole system to run based on the digital neuron substrate. Another work utilizes resonance energy transfer circuits (Wang et al. 2016), which exploits the probabilistic transfer 
Table 5. Comparison with Related Work

\begin{tabular}{|c|c|c|c|c|}
\hline Implementation & Kim et al. & Charoensak et al. & Shyu et al. & MRF BSS \\
\hline Algorithm & ICA & ICA & FastICA & MRF \\
\hline Sampling rate $(\mathrm{kHz})$ & 12 & 8 & 16 & 16 \\
\hline Platform & FPGA & FPGA & FPGA & FPGA, ASIC \\
\hline Frequency $(\mathrm{MHz})$ & 12.288 & 71.2 & 50 & 150,20 \\
\hline Latency (ms) & $\geq 10,000$ & 620 & 3 & $1.6,12$ \\
\hline Power $(\mathrm{mW})$ & 197.6 & $\mathrm{~N} / \mathrm{A}$ & $\mathrm{N} / \mathrm{A}$ & N/A, 70 \\
\hline
\end{tabular}

for energy between two optically active molecules. This method is not practical or cost-effective for real world systems, as it requires an external device on a non-CMOS fabric.

Another set of work implements sound source separation on hardware. Omitting all the work that does not provide any qualitative evaluation and hardware performance numbers such as frequency, size, and power, we have listed three relevant hardware implementations as shown in Table 5. All three previous implementations are ICA based; ICA used to be a commonly used algorithm for source separation and it is very parallelizable as it consists of many linear algebraic operations. Kim et al. (2003) consumes relatively low power (197.6mW), which is the power required to run two-channel source separation, but does it in more than 10 seconds. Charoensak and Sattar (2005) also has a long latency of 620ms. Shyu et al. (2008) has low latency, close to the latency of our design running at $150 \mathrm{MHz}$, but does not report any power numbers. Furthermore, it does not have any discussion of qualitative sound separation performance. All of the implementations mentioned in Table 5 do not satisfy our design constraints mentioned in Section 4.

While there has been much improvements in sound source separation using algorithms other than ICA, the new algorithms for source separation tend to be more complex and difficult to implement in hardware for practical uses, which is why we suspect the lack of more recent implementation of sound source separation in hardware. Our MRF-bases source separation uses Gibbs sampling inference, which is sequential in nature but is not the best for hardware acceleration. Furthermore, using EM for parameter estimation results in two nested iterative loops. A straightforward implementation of MRF would not have been possible to meet the practical mobile use constraints listed in Section 4. By incorporating the methods discussed in Section 5, such as reusing the previous processed sound frames and inference results, we can construct and update MRF on the fly, achieving real-time streaming source separation with low latency and power.

Furthermore, compared to ICA, MRF-based source separation has several advantages. First, ICA only works well for overdetermined cases when there are as many or more microphones than the sources in the mixture, whereas MRF works well with underdetermined mixing problems as well. While ICA describes only the likelihood distribution, MRF can incorporate prior information as well, which allows more robust source separation. The EM parameter estimation for updating hyperparameters of the MRF in real-time works well even with moving sound sources.

\section{CONCLUSION}

We presented a novel real-time and low-power streaming architecture for sound source separation using MRF and Gibbs sampling inference. Our implementation is capable of learning parameters for constructing MRF on the fly using EM, while performing MAP inference using Gibbs sampling to find the best binary mask label assignment. The dataflow architecture that reuses previous sound frames and inference results allows us to achieve real-time performance while producing satisfactory separation of sound sources. An FPGA implementation runs at $150 \mathrm{MHz}$ with $207 \mathrm{~KB}$ 
RAM and while achieving SDR up to $7.021 \mathrm{~dB}$ with only $1.6 \mathrm{~ms}$ latency, a $22 \times$ speed-up; it confirms real-time streaming feasibility. A $45 \mathrm{~nm}$ virtual ASIC design running at $20 \mathrm{MHz}$ requires fewer than 10 million gates while consuming $70 \mathrm{~mW}$, which is less than $2 \%$ of the ARM Cortex-A9 software reference design. Overall, we believe that our design shows that our MRF source separation architecture is viable for real-time streaming implementation of sound source separation for mobile phones.

\section{REFERENCES}

Jason D. Bakos. 2010. High-performance heterogeneous computing with the convey HC-1. Comput. Sci. Eng. 12, 6 (2010), $80-87$.

Nathan Binkert, Bradford Beckmann, Gabriel Black, Steven K. Reinhardt, Ali Saidi, Arkaprava Basu, Joel Hestness, Derek R. Hower, Tushar Krishna, Somayeh Sardashti, et al. 2011. The GEM5 simulator. ACM SIGARCH Comput. News 39, 2 (2011), 1-7.

S. T. Birchfield and R. Gangishetty. 2005. Acoustic localization by interaural level difference. In Proceedings of the IEEE International Conference on Acoustics, Speech, and Signal Processing (ICASSP'05), Vol. 4. 1109-1112. DOI : http:// dx.doi.org/10.1109/ICASSP.2005.1416207

Y. Y. Boykov and M. P. Jolly. 2001. Interactive graph cuts for optimal boundary \& region segmentation of objects in N-D images. In Proceedings of the E8th IEEE International Conference on Computer Vision (ICCV'01), Vol. 1. 105-112. DOI : http:// dx.doi.org/10.1109/ICCV.2001.937505

Tony M. Brewer. 2010. Instruction set innovations for the Convey HC-1 computer. IEEE Micro 30, 2 (2010), 70-79.

D. Campbell, K. Palomaki, and G. Brown. 2005. A Matlab simulation of "shoebox" room acoustics for use in research and teaching. Comput. Inf. Syst. 9, 3 (2005), 48.

J. F. Cardoso. 1998. Blind signal separation: Statistical principles. Proc. IEEE 86, 10 (1998), 2009-2025. DOI : http://dx.doi.org/ $10.1109 / 5.720250$

C. Charoensak and F. Sattar. 2005. A single-chip FPGA design for real-time ICA-based blind source separation algorithm. In Proceedings of the IEEE International Symposium on Circuits and Systems (ISCAS'05). 5822-5825. DOI : http://dx.doi. org/10.1109/ISCAS.2005.1465962

E. Colin Cherry. 1953. Some experiments on the recognition of speech, with one and with two ears. 7. Acoust. Soc. Am. 25, 5 (1953), 975-979.

David E. Culler. 1986. Dataflow architectures. Ann. Rev. Comput. Sci. 1, 1 (1986), 225-253.

S. Das, B. U. Pedroni, P. Merolla, J. Arthur, A. S. Cassidy, B. L. Jackson, D. Modha, G. Cauwenberghs, and K. Kreutz-Delgado. 2015. Gibbs sampling with low-power spiking digital neurons. In Proceedings of the 2015 IEEE International Symposium on Circuits and Systems (ISCAS'15). 2704-2707. DOI : http://dx.doi.org/10.1109/ISCAS.2015.7169244

Frank Dellaert. 2002. The Expectation Maximization Algorithm. Technical Report. Georgia Institute of Technology.

Arthur P. Dempster, Nan M. Laird, and Donald B. Rubin. 1977. Maximum likelihood from incomplete data via the EM algorithm. f. Roy. Stat. Soc. Ser. B (Methodol.) 39, 1 (1977), 1-38.

Jack B. Dennis and David P. Misunas. 1975. A preliminary architecture for a basic data-flow processor. ACM SIGARCH Comput. Archit. News 3, 126-132.

Jérémie Detrey and Florent de Dinechin. 2007. Parameterized floating-point logarithm and exponential functions for FPGAs. Microprocess. Microsyst. 31, 8 (2007), 537-545.

J. S. Garofolo, L. F. Lamel, W. M. Fisher, J. G. Fiscus, D. S. Pallett, N. L. Dahlgren, and V. Zue. 1993. TIMIT acoustic-phonetic continuous speech corpus. In Proceedings of the Linguistic Data Consortium.

S. Geman and D. Geman. 1984. Stochastic relaxation, Gibbs distributions, and the Bayesian restoration of images. IEEE Trans. Pattern Anal. Mach. Intell. 6 (1984), 721-741.

John R. Gurd, Chris C. Kirkham, and Ian Watson. 1985. The Manchester prototype dataflow computer. Commun. ACM 28, 1 (1985), 34-52.

Roberto Gutierrez and Javier Valls. 2011. Low cost hardware implementation of logarithm approximation. IEEE Trans. VLSI Syst. 19, 12 (2011), 2326-2330.

Kaiming He, Xiangyu Zhang, Shaoqing Ren, and Jian Sun. 2015. Delving deep into rectifiers: Surpassing human-level performance on ImageNet classification. In Proceedings of the IEEE International Conference on Computer Vision. 10261034.

Geoffrey E. Hinton, Simon Osindero, and Yee-Whye Teh. 2006. A fast learning algorithm for deep belief nets. Neural Comput. 18, 7 (2006), 1527-1554. DOI : http://dx.doi.org/10.1162/neco.2006.18.7.1527

Harri Holma and Antti Toskala. 2011. LTE for UMTS: Evolution to LTE-Advanced. John Wiley \& Sons.

Aapo Hyvärinen, Juha Karhunen, and Erkki Oja. 2004. Independent Component Analysis. Vol. 46. John Wiley \& Sons. 
Telecommunication Standardization Sector ITU. 2003. International telephone connections and circuits-general recommendations on the transmission quality for an entire international telephone connection. ITU-T Recommendation G.114. ITU-T.

Joerg Kappes, Bjoern Andres, Fred Hamprecht, Christoph Schnorr, Sebastian Nowozin, Dhruv Batra, Sungwoong Kim, Bernhard Kausler, Jan Lellmann, Nikos Komodakis, et al. 2013. A comparative study of modern inference techniques for discrete energy minimization problems. In Proceedings of the IEEE Conference on Computer Vision and Pattern Recognition. 1328-1335.

Chang-Min Kim, Hyung-Min Park, Taesu Kim, Yoon-Kyung Choi, and Soo-Young Lee. 2003. FPGA implementation of ICA algorithm for blind signal separation and adaptive noise canceling. IEEE Trans. Neur. Netw. 14, 5 (2003), 10381046.

M. Kim, P. Smaragdis, G. G. Ko, and R. A. Rutenbar. 2012. Stereophonic spectrogram segmentation using Markov random fields. In Proceedings of the 2012 IEEE International Workshop on Machine Learning for Signal Processing. 1-6. DOI : http:// dx.doi.org/10.1109/MLSP.2012.6349754

G. G. Ko and R. A. Rutenbar. 2017. A case study of machine learning hardware: Real-time source separation using Markov random fields via sampling-based inference. In Proceedings of the 2017 IEEE International Conference on Acoustics, Speech, and Signal Processing (ICASSP'17). 2477-2481. DOI : http://dx.doi.org/10.1109/ICASSP.2017.7952602

Daphne Koller and Nir Friedman. 2009. Probabilistic Graphical Models: Principles and Techniques. MIT Press.

Vladimir Kolmogorov. 2006. Convergent tree-reweighted message passing for energy minimization. IEEE Trans. Pattern Anal. Mach. Intell. 28, 10 (2006), 1568-1583. DOI : http://dx.doi.org/10.1109/TPAMI.2006.200

Alex Krizhevsky, Ilya Sutskever, and Geoffrey E. Hinton. 2012. ImageNet classification with deep convolutional neural networks. In Proceedings of the 25th International Conference on Neural Information Processing Systems, Volume 1 (NIPS'12). 1097-1105.

Sheng Li, Jung Ho Ahn, Richard D. Strong, Jay B. Brockman, Dean M. Tullsen, and Norman P. Jouppi. 2009. McPAT: An integrated power, area, and timing modeling framework for multicore and manycore architectures. In Proceedings of the 42nd Annual IEEE/ACM International Symposium on Microarchitecture. ACM, 469-480.

D. J. C. Mackay. 1998. Introduction to Monte Carlo Methods. Springer, Dordrecht, Netherlands, 175-204. DOI : http://dx.doi. org/10.1007/978-94-011-5014-9_7

Vikash K. Mansinghka, Eric M. Jonas, and Joshua B. Tenenbaum. 2008. Stochastic Digital Circuits for Probabilistic Inference. Technical Report MITCSAIL-TR 2069. MIT, Cambridge, MA.

Thomas Minka. 1998. Expectation-Maximization as Lower Bound Maximization. Retrieved from http://www.cse.psu.edu/ rtc12/CSE598G/papers/minka98expectationmaximization.pdf.

John N. Mitchell. 1962. Computer multiplication and division using binary logarithms. IRE Trans. Electr. Comput. 4 (1962), 512-517.

Radford M. Neal and Geoffrey E. Hinton. 1998. A view of the EM algorithm that justifies incremental, sparse, and other variants. In Learning in Graphical Models. Springer, 355-368.

Judea Pearl. 1988. Probabilistic Reasoning in Intelligent Systems: Networks of Plausible Inference. Morgan Kaufmann, San Francisco, CA.

Nicoleta Roman, DeLiang Wang, and Guy J. Brown. 2003. Speech segregation based on sound localization. F. Acoust. Soc. Am. 114, 4 (2003), 2236-2252.

Olga Russakovsky, Jia Deng, Hao Su, Jonathan Krause, Sanjeev Satheesh, Sean Ma, Zhiheng Huang, Andrej Karpathy, Aditya Khosla, Michael Bernstein, Alexander C. Berg, and Li Fei-Fei. 2015. ImageNet large scale visual recognition challenge. Int. F. Comput. Vis. 115, 3 (2015), 211-252. DOI : http://dx.doi.org/10.1007/s11263-015-0816-y

Daniel Scharstein and Richard Szeliski. 2002. A taxonomy and evaluation of dense two-frame stereo correspondence algorithms. Int. F. Comput. Vis. 47, 1-3 (2002), 7-42. DOI : http://dx.doi.org/10.1023/A:1014573219977

K.-K. Shyu, M.-H. Lee, Y.-T. Wu, and P.-L. Lee. 2008. Implementation of pipelined fastICA on FPGA for real-time blind source separation. IEEE Trans Neural Netw. 19, 6 (2008), 958-970. DOI : http://dx.doi.org/10.1109/TNN.2007.915115

Richard Szeliski, Ramin Zabih, Daniel Scharstein, Olga Veksler, Vladimir Kolmogorov, Aseem Agarwala, Marshall Tappen, and Carsten Rother. 2008. A comparative study of energy minimization methods for Markov random fields with smoothness-based priors. IEEE Trans. Pattern Anal. Mach. Intell. 30, 6 (2008), 1068-1080. DOI : http://dx.doi.org/10. 1109/TPAMI.2007.70844

Thomas E. Tkacik. 2003. A Hardware Random Number Generator. Springer, 450-453. DOI:http://dx.doi.org/10.1007/ 3-540-36400-5_32

E. Vincent, R. Gribonval, and C. Févotte. 2006. Performance measurement in blind audio source separation. IEEE Trans. Audio Speech Lang. Process. 14, 4 (2006), 1462-1469.

Chaohui Wang, Nikos Komodakis, and Nikos Paragios. 2013. Markov random field modeling, inference \& learning in computer vision \& image understanding: A survey. Comput. Vis. Image Understand. 117, 11 (2013), 1610-1627. 
S. Wang, X. Zhang, Y. Li, R. Bashizade, S. Yang, C. Dwyer, and A. R. Lebeck. 2016. Accelerating Markov random field inference using molecular optical Gibbs sampling units. In Proceedings of the 2016 ACM/IEEE 43rd Annual International Symposium on Computer Architecture (ISCA'16). 558-569. DOI : http://dx.doi.org/10.1109/ISCA.2016.55

O. Yilmaz and S. Rickard. 2004. Blind separation of speech mixtures via time-frequency masking. IEEE Trans. Sign. Process. 52, 7 (2004), 1830-1847. DOI : http://dx.doi.org/10.1109/TSP.2004.828896

Received March 2017; revised December 2017; accepted January 2018 